\title{
SOBRE UM CASO DE TUMOR DO RETRO.PERITONIO
}

\author{
observagão tomada no serviģo dos profs. RUBiāo \\ MEIRA E ALMEIDA PRADO GM 24-1-1924, PELOS INTERNOS \\ DOMINGOS LAROCCA E BONIFACIO DE CASTRO FHHO
}

Nome - A. M., 36 annos, Italiano. Procedencia - Tambahú.
Leito - 9.

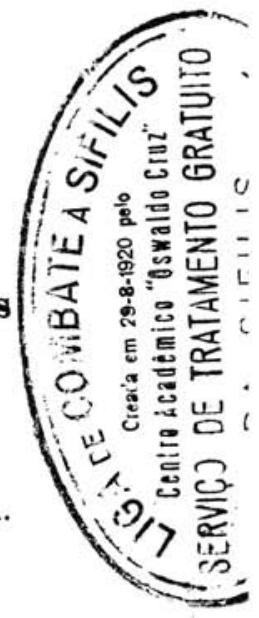

ANTECEDENTES HEREDITARIOS - Pae vivo e forte. Sua mâe falleceu victima de uma mordedura de cobra. Não teve aborto. Historia dos collateraes sem importancia. Tem seis irmãos sadios e robustos.

ANTECEDENTES PESSOAES - Em criança teve sarampo e nega as demais molestias peculiares á infancia: Teve ancylostomose aos 22 annos e grippe aos 30 . E' casado, teve seis filhos, tendo sua mulher abortado os tres primeiros e os restantes são vivos e fortes. Fuma muito; bebe moderadamente. Nega em absoluto passado venereo ou syphilitico.

HISTORIA ANTERIOR DA MOLESTIA ACTUAL - Refere o doente que aos dez annos de edade, ao levantar um peso, sentiu uma dôr na região escrotal e, minutos após, examinando com mais cuidado, talvez só então percebendo da sua existencia, notou uma saliencia no mesmo lugar da dôr, semelhante a um pequeno limão, tumor esse que se reduzia, desapparecendo, quando era impellido para o canal inguinal, permanecendo, porém, o testiculo sempre no mesmo lugar. Assim decorreram os annos sem nenhum outro accidente, salvo pequenos surtos dolorosos, até que de dois annos para cá sem motivos apparentes, o tumor começou a crescer com rapidez, assumindo em breve as proporções de um volumoso pepino, de consistencia petrea, liso, doloroso á pressão, exacerbando-se a dôr com a posição $\theta$ permanencia de pé, não mais se reduzindo e deixando de existir distincção com o testiculo, sendo então um tumor unico. Nesta occasião não apresentava outras perturbações, mantendo todas as funç̧ões e orgãos em perfeito estado, salvo pequenos calefrios nas crises dolorosas. Em 29 de Janeiro de 1923, resolveu internar-se na Santa Casa, dando entrada na 2." C. H. sendo operado em 4 de Fevereiro pelo Prof. Alves Lima. O caso do nosso doente consta da observação N. 3 do Dr. Washington Pares, publicado "in these" de Fevereiro de 1923, da qual extrahimos os seguintes trechos:

"Ao ser examinado notava-se o escroto asymetrico, um dos ledos augmentado de volume, o testiculo doente encobria completamente, por um augmento de volume, o testiculo são. A pelle do escroto 
encontrava-se tensa, de uma côr rosea, não adherente ás camadas profundas $\theta$ percebia-se muito bem a réde vascular superficial. 0 tumor tinha forma ovoidal, alongado verticalmente (testiculo de touro) com uma superficie regular, uniformemente lisa, de consistencia dura, opaco e doluroso á pressão; o testiculo e o epydidimo formavam um só blóco, $\Theta$ a vaginal não era prehensivel entre os dedos. O cordão espermatico era normal; não havia tumefacção, endurecimento, espessamento dos vasos lymphaticos, e não era doloroso, mesmo quando se comprimia entre os dedos. Os ganglios lombares não eram apreciaveis e, pelo toque retal, a prostata, ag vesiculas seminaes, etc. nada accusavam. O doente, objecto desta observação é um indiviỏuo de estatura alta, bem constituido, com paniculo adiposo e musculatura bem desenvolvidos. $O$ seu estado geral é optimo. O exame dos diversos apparelhos veiu attestar o estado de bom funccionamento em que elles se encontram.

Systema ganglionar - Ganglios inguinaes presentes.

Exames de laboratorio: Sangue: - Wassermann - negativo. Urina - Albumina, assucar, nihil.

Diagnostico - Neoplasma do testiculo.

Praticada a operação em 4 de Fevereiro é retirado um volumoso tumor que foi enviado ao Instituto Anatomo-Pathologico da Faculdade de Medicina, vindo com diagnostico de "Teratoma". Como o doente apresentasse um bom estado geral, e não fossem perceptiveis clinicamente metastases abdominaes, 15 dias após a primeira intervenção, com seu pleno consentimento, o doente é submettido a uma nova operação e pratica-se a castração simples sem exerese do pediculo lympho-ganglionar, porquanto não havia necessidade para tal - os lymphaticos do cordão e os ganglios lombares não tinham sido invadidos pelo tumor - já por ser o tumor de natureza benigna, e uma prova mais clara era dada pela grande porção do cordão espermatico que foi retirada e que, parecendo normal, em nada nos aconselhava a ir mais adiante". Dos exames anatomo-pathologicos, sómente damos noticia do exame microscopico - para o exame macroscopico e descripção, veja-se a these do Dr. Washington Pares, Fevereiro de 1923.

1. PEÇA - EXAME MICROSCOPICO - Não é possivel distinguir vestigios de qualquer orgão. Nos preparados percebemos variaß especies de tecidos dispostos desordenadamente. Numerosas formações glandulares revestidas por cellulas cylindricas, ás vezes com apparencia fusiforme, outras vezes estratificadas; suas luzes são irregulares e formam desenhos variados. Junto a ellas existem cavidades kysticas, desprovidas de epithelio e ás vezes reduzidas a uma camada de cellulas cubicas, claras, contendo uma substancia semelhante á gelatina. Essas formações estão circumdadas por varias especies de tecidos, predominando o tecido mucoso em algumas partes, em outras tecido fibroso $\theta$ em outras tecido cartilaginoso, o qual fórma verdadeiros nodulos. Além disso, é possivel distinguir algumas fibras de tecido muscular liso.

DIAGNOSTICO - Teratoma do testiculo".

2." PEÇA - EXAME MACROSCOPICO - "São encontrados restos de epydidimo, etc. Mais adiante diz o autor: - Do tumor que corresponde ao primeiro exame, não encontramos formações que a 


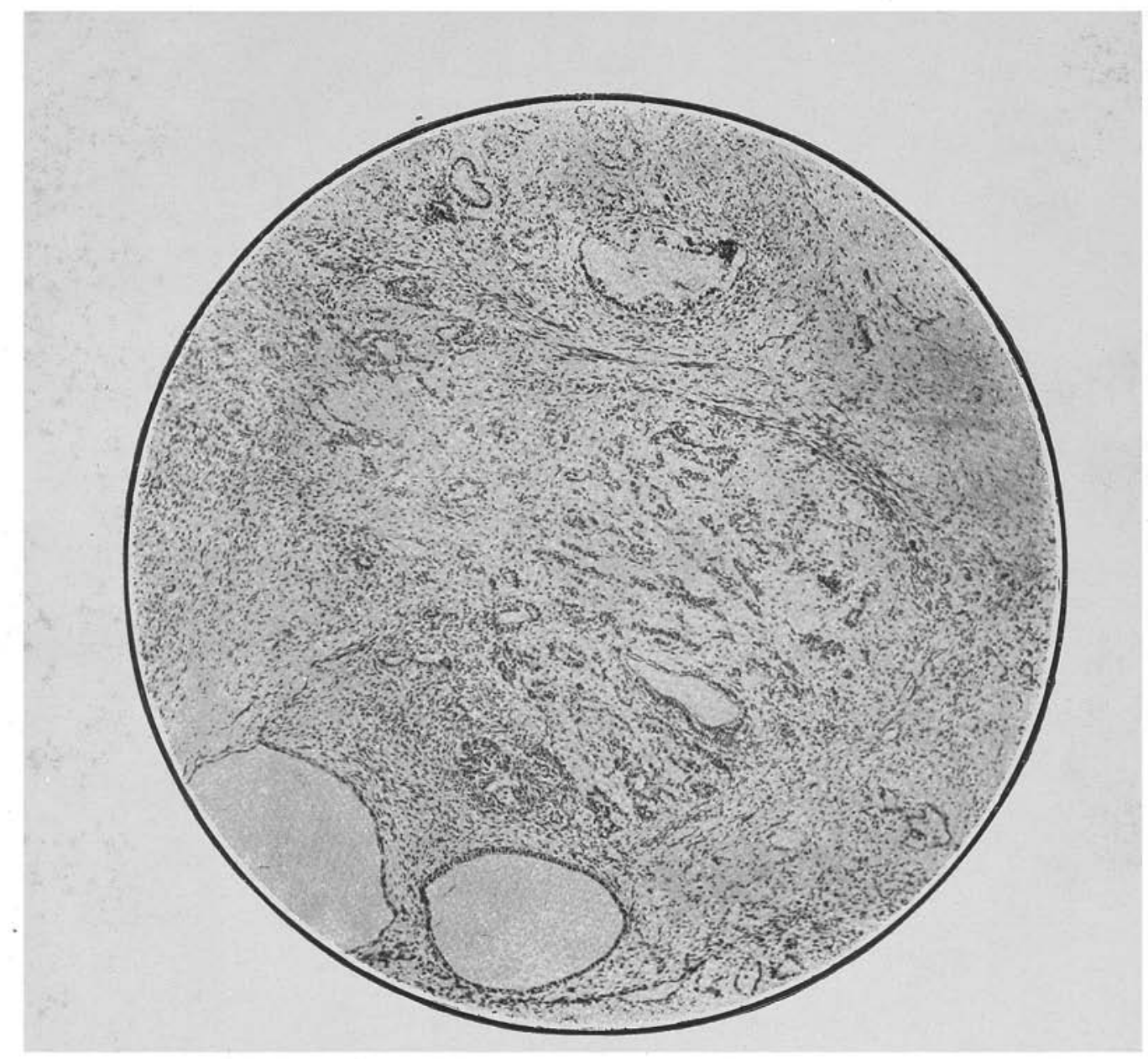

S-596-23 Object $16 \mathrm{MM}$ Ocul $5-55$ Diam. 
elle se assemelhem, porém, em algumas partes é interessante notar certas formações que se assemelham a villus placentarios, tendo geu centro um vaso, com estroma connectivo bastante frouxo, claro $\theta$ as cellulas que o revestem se assemelham perfeitamente com as camadas de Langhans. Em conjuncto, a peça parece formar uma parte da parede do tumor que não foi retirada na primeira operação e que apresenta ainda alguns restos tumoraes.

DIAGNOSTICO - Restos do testiculo, epydidimo e cordão espermatico. Hematoma do testiculo. Restos do tumor".

Da descripção detalhada do exame microscopico juntamos o seguinte desenho para elucídar e dar uma idéa nitida dos diversos tecidos e partes que o compõem:

\section{CORTE DA 1.* PEÇA (S.598-23)}

\section{(Veja-se desenho annexo)}

O doente teve alta curado em 18 de Março, após um periodo post-operatorio, sem accidentes (conforme se lê nos archivos da 2." C. H.). Decorreram-se assim 6 mezes, quando o doente começou a sentir pontadas frustras que comparou a agulhadas, intermittentes, localisadas ora no flanco direito, ora na região umbilical, desapparecendo ao cabo de dois dias. Ha um mez e meio foi surprehendido novamente pela mesma dôr, porém, desta vez mais intensa, localisada nas mesmas regiões, irradiando-se para a região lombar, onde era mais accentuada. Durante a noite, ás vezes a posição em decubito era impossivel e para conciliar o somno, o que conseguia poucas vezes, era obrigado a recostar-se ao leito. Dias após, a coloração das urinas ficou mais carregada, mas nunca turva ou hematurica. As miç̧ões que, sempre foram de 4 a 5 diurnas, uma a duas nocturnas, mantiveram-se sempre as mesmas com a molestia. Para acalmar as dôres, além do repouso, tomava frequentes purgativos e informa que durante a acção destes o peristaltismo era muito mais accentuado para o lado esquerdo do abdomen, ao passo que o direito se mantinha immovel como que endurecido. Em uma destas occasiões, paseando a mão com mais cuidado sobre a região umbilical e flanco direito, notou uma pequena tumefacção, resistente e dolorosa, que muito o impressionou. Ha 20 dias atrás, apćs pesados trabalhos, foi acommettido de semelhante dôr, mais violenta que as outras, parecendo, segundo a propria expressão do doente, "como uma cousa que o queimasse por dentro". Foi obrigado a acamar-se por alguns dias, porém, quasi sempre recostado. Aconselhado pelo clinico do lugar procurou novamente a Santa Casa, dando entrada em nossa Enfermaria em 16 de Janeiro do corrente anno.

\section{EXAME DO DOENTE}

EXAME PHYSICO - INSPECÇÃO - Individuo moço, de constituição ossea e muscular não muito desenvolvida. Typo longilineo ou esthenico não apresentando nenhuma dysmorphia. Habitus enteroptoticus. Acha-se bastante emmagrecido. Facies pallida. Paniculo adıposo escasso. Segmento cephalico normal. Pescoço fino, longo, projectado para frente, cartilagem thyroide saliente, membros su- 
periores finos, vasos visivels $\theta$ tensos. Nos membros inferiores apresenta ligeiro edema premalieolar. No escroto observamos a cicatriz operatoria. Thorax longo, estreito e chato. Angulo de Louis sallente - fossas supra-claviculares accentuadas, claviculas horizontaes, peitoraes atrophiados. Circumferencia thoraxica, ao nivel dos dois mamillos, $82 \mathrm{cms}$. Do lado direito do thorax notamos a presença de circulaç̃o collateral. Angulo de Charpy medindo cerca de $30^{\circ}$. Espaços intercostaes largos, visiveis e inclinados. Escapula alata. Não ha hetero-morphias thoraxicas que demonstrem qualquer affecção do thorax. Appendice xyphoide delgado e longo. Indice de Lenhoff

$$
\begin{array}{ll}
\text { distancia jugulo-pubica } & =54 \\
\text { menor circumferencia abdominal } & =70
\end{array} \times 100=77
$$

ABDOMEN - Pela simples inspecção observamos do lado direito, nas regiōes do flanco direito e umbilical, não attingindo a linha mediana, uma saliencia lisa, arredondada, immovel com os movimentos respiratorios, tornando menos accentuado o angulo costoilliaco. Os limites desapparecem gradativamente formando, com o rebordo costal direito, uma pequena depressão. Cicatriz umbilical na linha mediana, a $17 \mathrm{cms}$. acima do pubis e a igual distancia do appendice xyphoide. Tamhem notamos no abdomen a presença de circulação collateral do lado direito.

\section{EXAME DOS DIVERSOS APPARELHOS}

APPARELHO RESPISATORIO - INSPECCÃO - Encontramos os mesmos signaes descriptos acima. Dezenove movimentos respiratorios por minuto. Fhenomeno de Litten presente de ambos cs lados. Respiração do tyṇı costo-abdominal.

PALPAÇÃO - Fremito ligeiramente augmentado em todo o hemi-thorax direito.

PERCUSSÃO - Nada de importante nos informa. Excursão dos bordos pulmonares inferiores e posteriores de cerca de 4 a 5 cms.

AUSCULTA - Nada te importante.

APPARELHO CIRCULATORIO - INSPECÇÃO - Nada nos revela.

PALPAÇÃO - Ictus cordis no $5 .^{\circ}$ espaço para dentro da linha mamillar. Ausencia de tremito.

PERCUSSÃO - Area cardiaca não augmentada.

AUSCULTA - Bulhas cardiacas normaes com hyperphonese da segunda bulha ao nival do fóco aortico.

Pulso cheio e rythmado, 86 pulsações por minuto. Pressão sanguinea pelo Riva-Rocci, 13 maxima e 7,5 minima. Reflexo oculocardiaco, baixa para 66.

SYSTEMA NERVOSO - Reflexos cutaneos, osseos, tendinosos, luz, accommodação, etc. - normaes.

SYSTEMA GANGLIONAR - Ganglios palpaveis sómente nas regiôes inguinaes.

ABDOMEN - Cupola do figado, 2 dedos abaixo do mamillo. Bordo inferior, difficilmente palpavel. Altura, $14 \mathrm{cms}$. Baco não palpavel e não percutivel. Espaço de Traube - livre. 


\section{CARACTERES DO TUMOR}

Tumor de fórma quasí quadrangular. A principio bem paipavel, actualmente manifesta-se uma reacção dolorosa que não permitţ fazel-a profundamente. Bordos arredondados, com maior eixo dirigido de cima para baixo e da direita para a esquerda (16 cms.), com $12,5 \mathrm{cms}$. no sentido longitudinal e 12 no sentido horizontal. Podemos considerar 4 burdos: superior, inferior, externo e interno. Bordo inferior a $9 \mathrm{cms}$. acima do pubis. O superior a $10 \mathrm{cms}$. abaixo do appendice xyphoide. $O$ bordo interno não attinge a linha mediana e apresenta uma pequena reintrancia na sua parte media. A porção. inferior do bordo externo está a $3 \mathrm{cms}$. da crista illiaca.

SEDE - Está situado na porção direita do abdomen não ultrapassando a linha mediana. Occupa as regióes do flanco direito e umbilical na sua porção inferior.

PALPAÇÃO - Tumor de consistencia lenhosa, de superficie lisa, doloroso á pressão em toda a sua area, principalmente na porção interna do bordo inferio: que desperta dôr que se irradia para a urethra. Do seu bordo externo para a massa dos musculos sacrolombares a palpação é dolorosa, dando sensação nitida de resistencia. Entre o bordo superior e o rebordo costal, ha uma zona depressivel onde se percebe gargarejos. Em toda a sua extensão palpa-se o bordo inferior do figado, facto que demonstra a náo continuidade com a viscera. Na superficie do tumor percebe-se gargarejos, podendo-se, $€ m$ determinadas occasices, deslocar o collo transverso para baixo. Na linha mamillar o tumor entra em contacto com o rebordo costal, não se determinando pela palpação o limite entre ambos. A contracção dos musculos rectos determina o desapparecimento da massa tumoral. A posição genu-peitoral torna flacida a parede abdominal e obscurece-a. O tumor a principio apresentava um ligeiro grau de mobilıdade respiratoria no sentido axial. Actualmente acha-se immovel.

PERCUSSÃO - Observamos ligeiro tympavismo em toda a sua extensão e principalmente as nivel da zona que o separa do bordo inferior do figado, desspparecendo ao nivel da linha mamillar. A exploração do estomago feita com pós gazogenicos não deu deslccamento nem perda de relação com os bordos, mas exaggerou o tympanismo no sulco separativo com o figado. A insufflação do intestino grosso, por via rectall, á custa de uma sonda ligada á parede de Richardson, á medida que as alças se distendiam, ia-se perdendo progressivamente o contacto com o tumor, do bordo interno ao externo, até desapparecer completamente e só deixar perceber gargarejos, e exaggero do tympanismo pela percussão, sendo portanto posterior a relação com o tubo gastro-intestinai. A exploração da região lombar apresenta um grau de flacidez maior em relação á do lado opposto. A pesquiza dos pontos dolorosos renaes e uretericos (O costo-muscular, o sub-costal, o supra-intra-espinhoso, ureterico superior ou para-umbilical que se irradia para a urethra e ureterico medio) foi positiva. A percussão das massas sacro-lombares dá á direita som obscuro, massico, massicez lenhosa que continua além da borda externa desses musculos, dando então sensação de resistencia. Para o lado esquerdo, sub-massicez em relação ao direito e além da borda das massas sacro-lombares obtem-se um som tympanico de sonoridade intestinal. 
TOQUE RECTAL - Feito em 23-1-1924 pelo Dr. Cintra Gordinho. Vesiculas seminaes perceptiveis, mais á esquerda que á direita. Prostata normal. Nada se observa em relação á empola rectal. Em 24 de Fevereiro foi feito o catheterismo dos uretéres cystoscopia, pyelographia e radiographia da região (Dr. Raphael de Barros e C. Gordinho).

Resultado destes diversos exames: - Relatorio do Dr. Gordinho.

0 exame cystoscopico da bexiga nos mostra que esta se acha mais ou menos normal, não congestionada, vendo-se algumas columnas normaes. O orificio ureteral direito parece sensivelmente atresiado, dando a impressão de uma fenda quasi invisivel. 0 orificio ureteral esquerdo é normal. Feito o catheterismo ureteral direito, a sonda penetra o meato com difficuldade e mais ou menos a $10 \mathrm{cms}$. acima deste, encontrei uma seria resistencia ao fazer progredir a sonda. A $20 \mathrm{cms}$. mais ou menos, cahi em pleno bacinete. As ejaculeções não appareceram immediatamente, sóment a 4 minutos após.

PYELOGRAPHIA - (Chapas 141 A e B) - Em seguida injectamos uma solução de bromureto de potassio á $25 \%$ para se proceder á pyelographia. 0 bacinete se mostra sensivelmente dilatado, pois o doente só accusa dôr após injecção de 15 c.c. de líquido, assim tambem como é bastante irregular e distendido, com a extremidade superior do ureter francamente dilatada, o que esclarece a situação do tumor, dilatação que se encontra acima da parte compressora. Nota-se que durante $o$ acto do catheterismo foi encontrada uma seria resistencia na penetração da sonda o que vem explicar facilmente essa dilatação concomitante da urethra e do bacinete produzido pelo dito tumor, explicando ainda a athresia do meato uretral direito verificado pela cystoscopia. Foi feita ume radiographia (chapa $141 \mathrm{C}$ ) localizando o centro do tumor, que nada revelou da sua presença, sendo transparente, situado muito abaixo dos calices injectados com bromureto de potassio, parecendo não existir dependencia com o rim. A sonda foi deixada no ureter para se recolher a urina do rim direito; infelizmente o doente não resistindo á dôr retirou-a após meia hora. A urina aproveitada do rím direito foi de 3 c.c. e a da bexiga, após a retirada da sonda ureteral foi de cerca de 50 c.c."

o exame feito no laboratorio central revelou em ambas as urinas:

Albumina - contém muita.

Hemoglobina - contérn (traumatismo operatorio).

Pesquiza de cellulas tumoraes pelo catheterismo - negativa.

EXAME MICROSCOPICO - Grande quantidade de hematias (traumatismo operatorio) e leucocytos. Rarissimos cylindroides e cylindros hyalinos. Exame de urina antes do catheterismo-21-1-1924.

Albumina - traços.

Reacção de Weber - negativa.

Pesquiza de cellulas tumoraes - negativ3.

Sedimento - Raros laucocytos, cellulas de descamação da bexiga.

Reacção de Wassermann - negativa.

Exame de escarro - negativo.

Contagem de globulos:

Globulos vermelhos - 4.585 .400

Globulos hrancos - 4.600 por m.m.

Contagem especifica:

Neutrophilos - $825 \%$ n. $67 \%$ 


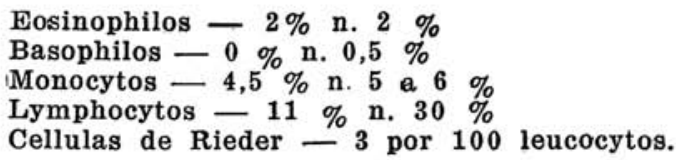

ESTADO ACTUAL - Até a presente data (12 de Fevereiro) o estado do nosso doente tem-se mantido relativamente bom, apesar do crescimento rapido e extraordinario da massa tumoral. Acha-se um tanto emmagrecido, mas ainda conserva um optimo estado psychico, tendo esperança de que seus males regridam com o tratamento puramente paliativo a que actualmente está se submettendo - arsenicaes, quina, etc. - Após o catheterismo, a febre que até então não havia sido observada, começou a manifestar-se, as urinas tornaram-se turvas e declarou-se uma pyelite que foi facilmente combatida com oito injecções de urotropina. Em $1 .^{\circ}$ de Fevereiro restabeleceu-se novamente a temperatura e o exame feito em 8-2-1924 mostrou-nos algumas alterações que provavelmente são restos do traumatismo determinado pelo catheterismo. Manifesta uma anorexia intermittente, o edema premalleolar acha-se bestante accentuado. $O$ numero de micções é, em média, de 4 a 5 diurnas e 5 nocturnas. Pela simples inspecção do tumor notamos seu notavel crescimento, chegando até a linha mediana. Em dadas occasiões percebem-se movimentos intestinaes. A região lombar direita acha-se mais abahulada e a curva do quadril menos accentuada. No lado esquerdo do abdomen existe circủlação collateral em communicação com a thoraxica. Pela palpação ha augmento consideravel de volume, ultrapassando de dois dedos a linha mediana. Percebem-se gargarejos e palpa-se o cóllo. Os pontos dolorosos desapparecem, accusando sómente pequenas dores frustras que não o incommodam muito. Immobilidade completa. Pela percussão a zona tympanica acima do tumor está ligeiramente diminuida, pois, o crescimento manifesta-se sobretudo para a parte inferior, posterior e linha mediana. Actualmente pela palpação observam-se ganglios na região axillar e, facto importante, o ganglio de Troisier - duro, e do tamanho de ume. avellã.

EXAMES DE LABORATORIO:

Urina - 27-1-1924.

Weber - negativo.

Albumina - traços.

Sedimento - nada de anormal.

Urina - 30-1-1924.

Albumina - traços.

Sedimento - cylindros hyalinos raros - pús em pequena quantídade.

Raras hematias.

Urina - 1-2-1924.

Albumina - regular quantidede.

Sedimento - raros sy!indros hyalinos, augmento regular de leucocytos e raras hematias.

Exame de urina - 8-2-1924.

Albumina - quantidade regular.

Sedimento - poucas hematias, leucocytos e rarissimos cylindros hyalinos.

Antes de entrarmos no diagnostico, vejamos algumas considerações sobre os teratomas do testiculo. 
Os teratomas do testiculo são de observação não muito frequente. São constituidos pelos tecidos que entram na constituição dos mais teratomas. A sua constituição é muito complexa; mau grado essa complexidade possuem caracteres communs que permittem englobal-os sobre a mesma denominação: são dysemóriomas, de tecidos multiplos, dos quaes alguns, mantidos ou tornados indefinidamente ferteis, crescem progressivamente. O crescimento dos teratomas é puramente local e não infiltrativo. As cellulas dos teratomas são como que enxertos embryonarios e dão tumores de crescimento limitado e que uma vez alcançado o termo de sua evolução, são constituidos por tecidos adultos. No emtanto, essa fertilidade a termo das cellulas embryonarias póde transformar-se em uma fertilidade indefinida, conferindo-lhes assim um caracter neoplasico. Em resumo, os dysembryomas não são como outras heterotopias, neopiasmas, mas podem tornar-se neoplasmas. Para o caso dos teratomas testiculares falha a regra seguida pelos dysembryomas puros, que é o crescimento limitado. A sua evolução nos é dada pela seguinte proposição de Masson: Na maior parte dos casos esses tumores têm um crescimento local, lento e continuo e em regra a este succede um crescimento rapido invasor e seguido de generalisação. Em outros termos, a maior parte dos dysembryomas testiculares comporta-se no começo como neoplasmas benignos e termina sua evolução como cancros. A fertilidade se manifesta sobretudo nas massas epitheliaes de origem wolfiana. Justamente no presente caso, uma cousa que chama logo a attenção, desde que se tenha estudado uma série de córtes do tumor, é a quantidade de tecido epithelial wolfiano, que assumindo fórma de acinos glandulares, parece penetrar infiltrativamente na massa restante do tumor. Trata-se effectivamente de um caso em que o elemento epithelial adquiriu uma fertilidade bastante accentuada. Os teratomas do testiculo pódem apresentar varios tecidos, taes como: ectoderma chorial, epiderme e derivados, neuro-epithelios, endoderma, tecidos wolfianos, tecidos mesenchymatosos. No caso em questão encontram-se sómente as duas uitimas categorias de tecidos: wclfianos e mesenchymatosos. A experiencia demonstra que o tumor mixto não é em si canceroso mas que quasi sempre se torna. A observação ensina que são os epithelíos do embryoma que dão origem ao cancro. Estamos, portanto, autorizados a dizer que foi o elemento epithelial wolfiano o responsavel pela cancerisação do tumor que nos occupa. As metastases tumoraes estabelecem-se como de regra para os ganglios lombo-aorticos homo-lateraes. Os symptomas urinarios que porventura apparecem poderão ser explicados por uma compressão exercida pelo tumor sobre o pediculo renal, sobre o proprio rim, ou, o que é menos provavel, por uma invasão do processo neoplasico sobre o rim.

\section{CONCLUSõES}

1) Os teratomas dos testiculos são tumores benignos.

2) Pódem degenerar, após algum tempo, em tumores malignos, pelo desenvolvimento excessivo de um dos tecidos componentes, que assume um caracter infiltrativo, e que se generaliza dando metastases.

3) Dos tecidos componentes do teratoma, o que mais facilmente se canceriza é o epithelial.

4) Dos tecidos epitheliaes dos embryomas testiculares, o que mais commumente sofîre degeneração maligna é o wolfiano. 
5) As metasteses se observam sempre para os ganglios retroperitoneaes homolateraes.

DIAGNOSTICO:

Pela historia anterior, evolução, provas de insufflação, etc., já de antemão haviamos pensado em um tumor dos ganglios retro-peritoneaes, facto que vem se fortalecer com a ajuda dos diversos exames propedeuticos e provas de pyelo e radiographia procedidas. A presença do ganglio de Troisier por si só fala a favor da existencia de um tumor abdominal e ainda mais da sua natureza - carcinomatosa, facto que se acha em perfeita relação com o desenvolvimento, e caracter histologico do primitivo tumor testicular.

DEGENERAÇÃO EPITHELIOMATOSA DO PRIMITIVO TERATOMA TESTICULAR COM METASTASE PARA OS GANGLIOS RETRO-PERITONEAES.

TUMOR DOS GANGLIOS RETRO-PERITONEAES (Carcinoma).

São Paulo, 14 de Fevereiro, 1924.

Os Internos:

BONIFACIO DE CASTRO FILHO

DOMINGOS LAROCCA

\section{LABORATORIO DE MICROSCOPIA}

E

ANALYSES CLINICAS

\section{Dr. Altino Anfunes}

RUA DO CARMO N. 11

Telepho. 2463 (Central)

SÃo PAUlo 